\title{
ARTIGO
}

do1 https://doi.org/10.22481/praxisedu.v16i41.6415

\section{CARACTERÍSTICAS DAS PESQUISAS QUE INVESTIGARAM A FORMAÇÃO DE PROFESSORES NO BRASIL POR MEIO DE COMUNIDADES DE PRÁTICA}

\author{
CHARACTERISTICS OF RESEARCH THAT INVESTIGATED TEACHER TRAINING \\ IN BRAZIL THROUGH PRACTICE COMMUNITIES
}

\author{
CARACTERÍSTICAS DE LAS INVESTIGACIONES QUE INVESTIGARON LA \\ FORMACIÓN DE PROFESORES EN BRASIL A TRAVÉS DE COMUNIDADES \\ PRÁCTICAS
}

Greice Maiara Denker

Universidade Regional de Blumenau - Brasil

Camila Galvan
Universidade Regional de Blumenau - Brasil

Rita Buzzi Rausch

Universidade Regional de Blumenau - Brasil

Universidade da Região de Joinville - Brasil

\begin{abstract}
Resumo: Este artigo objetiva compreender características das pesquisas em educação que investigaram a formação de professores no Brasil por meio de comunidades de prática. Foram analisadas pesquisas publicadas no banco de teses e dissertações da capes nos últimos dez anos. Trata-se de uma pesquisa qualitativa de revisão do tipo Estado da arte. Nesta perspectiva e por meio dos descritores: comunidade de prática, formação de professores e formação docente resultaram 21 pesquisas. Destas, analisamos as principais temáticas abordadas, os objetivos, o suporte teórico e os resultados obtidos. Observamos que as principais temáticas envolveram formação inicial e continuada de professores sendo a comunidade de prática um processo utilizado com foco colaborativo, integrando as tecnologias da informação e comunicação. Os objetivos das pesquisas classificam-se em sua maioria como propositivos e descritivos. Dentre os autores, Wenger (2001) ganha destaque quando se trata de Comunidade de Prática, Fiorentini (2014), Gatti (2010), Imbernón (2009) e Nóvoa (2009) ao tratar da formação de professores e Almeida (2008) foi o mais citado quando relacionado às TICs na Educação. Inferimos que propostas atuais acerca da formação de professores indicam contribuições positivas quando envolvem comunidades de prática, por traduzir-se em estratégia de desenvolvimento profissional docente eficaz e moderna que ressignificam os espaços de formação e a própria identidade docente. As pesquisas indicam ainda que o uso da comunidade de prática como um instrumento para o desenvolvimento profissional docente abre portas para Tecnologias de Comunicação e informação, inovando propostas que valorizam e estimulam o compartilhamento entre profissionais.
\end{abstract}

Palavras-chave: Comunidades de prática. Formação de professores. Pesquisas em educação. 


\begin{abstract}
This article aims to understand characteristics of research in education that investigated teacher education in Brazil through communities of practice. Research published in the capes thesis and dissertation database over the past ten years was analyzed. This is a qualitative, state-of-the-art review research. In this perspective and through the descriptors: Community of practice, teacher training and teacher training resulted in 21 surveys. Of these, we analyze the main themes addressed, the objectives, the theoretical support and the results obtained. We observed that the main themes involved initial and continuing teacher training, the community of practice being a process used with a collaborative focus, integrating information and communication technologies. Most research objectives are classified as propositional, and descriptive. Among the authors, Wenger (2001) stands out when it comes to the Community of Practice, Fiorentini (2014), Gatti (2010), Imbernón (2009) and Nóvoa (2009) when dealing with teacher training and Almeida (2008) was the most cited when related to ICT in Education. We infer that current proposals about teacher training indicate positive contributions when they involve communities of practice, as it translates into an effective and modern teacher professional development strategy that resignifies the training spaces and the teaching identity itself. Research also indicates that the use of the community of practice as an instrument for the professional development of teachers opens doors to Communication and Information Technologies, innovating proposals that value and encourage sharing among professionals.
\end{abstract}

Keywords: Communities of practice. Education research. Teacher education.

Resumen: Este artículo tiene como objetivo comprender características de la investigación en educación acerca de la formación del profesorado en Brasil mediante comunidades de práctica. Se analizó la investigación publicada en la base de datos tesis y disertaciones de Capes en los últimos diez años. Es una investigación de revisión cualitativa. En esta perspectiva y con los descriptores: comunidad de práctica, y la formación del profesorado resultó 21 encuestas. De estos, analizamos los temas abordados, objetivos, el soporte teórico y los resultados obtenidos. Observamos que los temas principales involucraban la capacitación inicial y continua de los docentes, siendo la comunidad de práctica un proceso utilizado con un enfoque colaborativo, integrando tecnologías de información y comunicación. La mayoría de los objetivos de investigación se clasifican como proposicionales y descriptivos. Entre los autores, Wenger (2001) se destaca cuando se trata de la Comunidad de Práctica, Fiorentini (2014), Gatti (2010), Imbernón (2009) y Nóvoa (2009) cuando se trata de la formación docente y Almeida (2008) fue el más citado cuando se relaciona con las TIC en educación. Inferimos que las propuestas actuales sobre la formación docente indican contribuciones positivas cuando involucran comunidades de práctica, ya que se traduce en una estrategia de desarrollo profesional docente eficaz y moderna que resignifica los espacios de formación y la identidad docente en sí. Indica también que el uso de comunidad de práctica como instrumento para el desarrollo profesional de los docentes, abre las puertas a las Tecnologías y para propuestas innovadoras que valoran el intercambio entre profesionales.

Palabras clave: Comunidades de práctica. Investigación educativa. Formación docente.

\title{
1. Introdução
}

Este artigo aborda as principais características das pesquisas em educação publicadas no banco de teses e dissertações da CAPES relacionadas às Comunidades de Prática na formação de professores realizadas nos últimos dez anos - 2008 a 2018 - no Brasil. O foco é identificar quais as principais temáticas pesquisadas, os objetivos de cada pesquisa, os 
principais autores utilizados, bem como os resultados obtidos. Trata-se de uma pesquisa qualitativa de revisão (ROMANOWSKI, 2006).

Essa pesquisa está inserida no Programa de Pós Graduação em Educação da Universidade Regional de Blumenau - FURB e vinculada ao GPFORPE - Grupo de Pesquisa em Formação de Professores e Práticas Educativas que tem por objetivo investigar a formação de professores e práticas educativas em contextos formais e não formais, considerando processos que envolvem o desenvolvimento profissional docente, a fim de elaborar conhecimentos científicos que contribuam para a qualificação dos processos de ensinar e aprender (FURB, 2019).

O interesse pelas Comunidades de Prática como alternativa para a formação de professores se dá por concordarmos com as ideias de Wenger (2010) de que a aprendizagem envolve relações sociais, abrangendo neste contexto, muito mais do que a relação mestre e estudante. As CoPs, como são chamadas as Comunidades de Prática, são formadas por profissionais que se reúnem de maneira voluntária e que possuem um objetivo em comum (WENGER, 2015). Trocam conhecimentos, experiências e informações de maneira colaborativa e compartilhada.

Compreendemos que as pessoas são os recursos mais valiosos quando se trata de Educação. Através das relações são gerados aprendizados e experiências que vão além das previstas ou esperadas. As relações durante os processos de ensino e aprendizagem são complexas e únicas, tendo interferência do meio social, pessoal e organizacional que os sujeitos estão inseridos. Nesse sentido, torna-se essencial levar em consideração as experiências e conhecimentos gerados pelos atores durante suas práticas. Concomitante a isso, pretendemos identificar pesquisas que envolvem as CoPs na área da Educação. Dialogamos com autores como Francisco Imbernón (2011); António Nóvoa (1992); Wenger (2015), bem como, com documentos que definem diretrizes para a formação inicial e continuada de professores, onde consideram que a ação docente esteja composta por dimensões de abrangência técnica, política, ética e estética. Portanto, a formação deve ser permanente, favorecendo ao profissional do magistério domínio e manejo dos conteúdos e metodologias, autonomia para tomada de decisão, inovações, tecnologia, conhecimento científico para aperfeiçoar a atuação e visão profissional.

É necessário, pois, perceber que existe conhecimento pedagógico especializado que se une à prática, possibilitando que as formações tenham como ponto de partida situações problemáticas da instituição educativa, inserindo processos de pesquisa para realizar uma ação, o que Imbernón (2011) chama de "inovação a partir de dentro". Neste sentido, visualizamos a 
Comunidade de Prática como uma alternativa que permite realizar a formação inicial e continuada, como é indicada pelos autores pesquisadores da área da Educação.

Diante disso, a pergunta de pesquisa que norteou a investigação foi: quais as características das pesquisas em educação do Brasil publicadas no banco de teses e dissertações da CAPES nos últimos dez anos (2008 - 2018), no campo da formação de professores, relacionadas às comunidades de prática? Este artigo tem como objetivo compreender as características das pesquisas que investigaram a formação de professores no Brasil por meio de comunidades de prática.

\section{Comunidade de prática na formação de professores}

A criação do termo Comunidade de Prática $(\mathrm{CoP})$ foi evidenciado por Etienne Wenger (2015) e, podemos ressaltar que em síntese, esse termo é usado para designar um grupo que se reúne com o objetivo de discutir determinado assunto por interesse próprio. Nesses encontros as pessoas trocam experiências e vivências com interesse comum ao aprendizado e à prática do que foi aprendido.

Embora o fenômeno seja antigo, o termo é relativamente recente, sendo utilizado por diversas organizações como alternativa de desenvolvimento profissional. No entanto, é preciso ter cautela, pois nem tudo que é chamado de comunidade, consiste em comunidade de prática, pois para ser definida como uma $\mathrm{CoP}$, é necessário alguns critérios como: domínio compartilhado de interesses no qual a competência é valorizada coletivamente, comunidade que constrói relacionamentos em que uns aprendem com os outros e prática compartilhada (WENGER et al, 2002).

A origem do termo se deu com os cientistas sociais e antropólogos Jean Lave e Etienne Wenger. A construção desse conceito se dá com base na aprendizagem e suas dimensões, podendo ser entendida como um sistema de aprendizagem social. Os cientistas sociais defendem que em uma $\mathrm{CoP}$ a responsabilidade pela gestão do conhecimento se torna coletiva, o que cria uma ligação entre conhecimento e desempenho e podem criar conexões que vão além de fronteiras organizacionais ou geográficas, podendo contar, inclusive, com instrumentos tecnológicos para sua implementação.

A criação das CoPs chama a atenção no mundo acadêmico e no mundo organizacional, pois aprender de forma coletiva e praticar aquilo que se aprendeu, amplia as oportunidades no atual cenário competitivo que vivemos. Na afirmação de Wenger (1998), nós prestamos atenção 
naquilo que esperamos ver, ouvimos aquilo que encontra espaço em nosso entendimento e agimos de acordo com nossas visões de mundo. Dessa maneira, as CoPs possibilitam o compartilhamento dessas visões, ampliando trocas, discussões e o desenvolvimento coletivo.

O Ministério da Educação, através da Resolução n. ${ }^{\circ}$ 2, de 1. . de julho de 2015 define as diretrizes curriculares nacionais para a formação inicial de professores em nível superior e para a formação continuada. O documento é pautado em princípios que norteiam a base comum nacional para a formação, conforme explanado:

Considerando os princípios que norteiam a base comum nacional para a formação inicial e continuada, tais como: a) sólida formação teórica e interdisciplinar; b) unidade teoria-prática; c) trabalho coletivo e interdisciplinar; d) compromisso social e valorização do profissional da educação; e) gestão democrática; f) avaliação e regulação dos cursos de formação;

Fica claro no documento que há importância na formação coletiva, colaborativa e que valorize as relações teoria-prática, bem como incentiva a articulação entre a graduação e a pósgraduação, fortalecendo o aprimoramento do profissional do magistério. Essa fala reafirma-se com as novas diretrizes, datadas do ano de 2019 que considera ainda, as diretrizes da BNCC Base Nacional Comum Curricular, sugerindo que a formação continuada seja entendida como componente essencial da profissionalização docente, devendo integrar-se ao cotidiano da instituição educativa e considerar os diferentes saberes e a experiência docente, e ainda, a compreensão dos docentes como agentes formativos de conhecimento e cultura e, como tal, da necessidade de seu acesso permanente a conhecimentos, informações, vivências e atualização culturais (BRASIL, 2019).

Os princípios que norteiam a formação visam o atendimento às especificidades dos profissionais nas diferentes etapas e modalidades de educação básica, observando as normas específicas definidas pelo Conselho Nacional de Educação (CNE). Considera que a ação docente esteja composta por dimensões da abrangência técnica, política, ética e estética, portanto a formação continuada deve favorecer ao profissional de magistério domínio e manejo dos conteúdos e metodologias, autonomia para tomada de decisão, inovações, tecnologia, conhecimento científico para aperfeiçoar a atuação e visão profissional.

Em seu Art. 16, inciso III, a resolução enfatiza a importância e "respeito ao protagonismo do professor e a um espaço-tempo que lhe permita refletir criticamente e aperfeiçoar sua prática”. (BRASIL, 2015). Considera ainda que, de acordo com a legislação, a formação continuada deve envolver "atividades formativas organizadas pelos sistemas, redes e 
instituições de educação básica incluindo desenvolvimento de projetos, inovações pedagógicas, entre outros" (BRASIL, 2015).

Pensando nesses processos, Imbernón (2011, p.41-42), afirma que:

O processo de formação deve dotar os professores de conhecimentos, habilidades e atitudes para desenvolver profissionais reflexivos ou investigadores. Nesta linha, o eixo fundamental do currículo de formação do professor é o desenvolvimento da capacidade de refletir sobre a própria prática docente, com o objetivo de aprender a interpretar, compreender e refletir sobre a realidade social e à docência.

Neste sentido, concordamos com o autor Wenger (2015) de que a aprendizagem envolve relações sociais, abrangendo neste contexto, muito mais do que a relação professor e estudante. Complementamos com as ideias do autor Nóvoa (1992, p. 14), que trata da formação continuada de professores abrangendo as relações sociais por meio da criação de redes.

É importante a criação de redes de (auto)formação participada, que permitam compreender a globalidade do sujeito, assumindo a formação como um processo interactivo e dinâmico. A troca de experiências e a partilha de saberes consolidam espaços de formação mútua, nos quais cada professor é chamado a desempenhar, simultaneamente, o papel de formador e de formando.

Wenger (2015) contribui ainda, refletindo sobre o espaço da escola e como este está interligado a outros contextos mais amplos, trazendo uma reflexão sobre a função social da escola e a função da aprendizagem para além dos espaços materiais de uma sala de aula.

Nesta perspectiva, a escola não é o locus privilegiado da aprendizagem. Não é um mundo fechado e autocontido, no qual os alunos adquirem conhecimento para ser aplicado fora, mas parte de um sistema de aprendizagem mais amplo. A classe não é o principal evento de aprendizado. É a vida em si que é o principal evento de aprendizado. Escolas, salas de aula e sessões de treinamento ainda têm um papel a desempenhar nessa visão, mas precisam estar a serviço da aprendizagem que acontece no mundo. (WENGER, 2015, p. 5).

Desta forma, visualizamos a Comunidade de Prática como uma alternativa que permite realizar a formação inicial e continuada, assim como indicada pelos autores pesquisadores e diretrizes da área da Educação por ter a possibilidade de contribuir para inserir a escola na sociedade de uma maneira mais ativa e reafirmar seu papel social, corroborando com a ideia de que a escola não deve estar sozinha nesse processo.

Para ser considerada uma Comunidade de Prática é preciso que haja informalidade, o que não significa desorganização e deve-se objetivar a resolução de problemas, portanto é mais 
que um grupo de aprendizes, são pessoas compromissadas em agregar as melhores práticas (WENGER, McDERMOTT, SNYDER, 2002). Podem ser grandes ou pequenas, presenciais ou virtuais, locais ou não e permitem que os praticantes gerenciem o conhecimento que precisam, criem conexões entre aprendizagem e desempenho e não necessitam ter restrições geográficas (WENGER, 2002).

Cultivar uma CoP não é tarefa fácil. Wenger (2002) defende que é preciso manter a essência de uma CoP desde a sua criação e definir um contexto estratégico, identificando problemas, articulando propostas e as necessidades de conhecimento. É possível identificar cinco estágios de desenvolvimento de uma comunidade de prática, sendo elas: potencial, união, madura, ativa e dispersa. Cada um desses estágios conta com princípios fundamentais, dentre eles podemos destacar: desenhar uma CoP pensando na sua evolução, manter o diálogo sobre as perspectivas, contemplar diferentes níveis de participação, desenvolver espaços públicos e privados para a comunidade, dar ênfase no valor que tem uma $\mathrm{CoP}$, combinar familiaridade e estimulação e criar um ritmo (WENGER et al, 2002).

\section{Metodologia}

Essa pesquisa identifica-se como uma pesquisa qualitativa, pois busca compreender as características das pesquisas que envolveram Comunidades de Práticas na formação de professores. Caracteriza-se também como uma pesquisa de revisão, por identificar e categorizar a produção científica dentro do período de dez anos, que abrange a temática selecionada. De acordo com Vosgerau e Romanowski (2014, p.167):

Os estudos de revisão consistem em organizar, esclarecer e resumir as principais obras existentes, bem como fornecer citações completas abrangendo o espectro de literatura relevante em uma área. As revisões de literatura podem apresentar uma revisão para fornecer um panorama histórico sobre um tema ou assunto considerando as publicações em um campo.

A escolha pela pesquisa qualitativa do tipo revisão se deu, uma vez que, há a importância de verificar qual a totalidade de estudos e pesquisas acerca da formação de professores e comunidades de prática, pois conforme Romanowski (2006), há a necessidade de um mapeamento que desvende e examine o conhecimento já elaborado e aponte os enfoques, os temas mais pesquisados e as lacunas existentes. Com a tecnologia, o acesso aos registros de pesquisas e às informações, se dá de maneira muito rápida, por isso Vosgerau e Romanowski 
(2014) afirmam que a análise do campo investigativo é fundamental neste tempo de intensas mudanças associadas aos avanços crescentes da ciência e da tecnologia. Dito isso, concordamos quando a autora afirma que uma pesquisa de revisão possibilita ainda, uma visão geral do que vem sendo produzido na área e uma ordenação que permite aos interessados perceberem a evolução das pesquisas, bem como suas características e foco, além de identificar as lacunas ainda existentes.

Para o levantamento das pesquisas foi utilizado o acesso ao banco de teses e dissertações da CAPES e realizado uma busca, delimitando o tempo, de 2008 a 2018, utilizando os descritores: "formação docente" AND “comunidade de prática" que resultou em 16 teses e/ou dissertações. Essa busca foi realizada durante o mês de agosto de 2019.

Em seguida, realizamos uma nova busca utilizando os descritores: "Comunidade de prática" AND "formação de professores", refinando a pesquisa com o filtro área do conhecimento, selecionando Educação, que apresentou 7 resultados.

Verificando os resultados obtidos, percebemos que dois trabalhos de pesquisa aparecem repetidos nas buscas, sendo assim, a análise do conteúdo abrangeu 21 trabalhos, conforme apresentados no quadro 1 .

Quadro 1- Pesquisas selecionadas

\begin{tabular}{|c|c|c|c|c|}
\hline ANO & TÍTULO & AUTOR & PESQUISA & PPGE \\
\hline$\stackrel{\circ}{\circ}$ & $\begin{array}{l}\text { Comunidades de prática na } \\
\text { formação docente: aprendendo a } \\
\text { usar ferramenta da web } 2.0\end{array}$ & $\begin{array}{l}\text { Bohn, Vanessa } \\
\text { Cristiane } \\
\text { Rodrigues }\end{array}$ & $\begin{array}{l}\text { Dissertação } \\
\text { D1 }\end{array}$ & UFMG \\
\hline 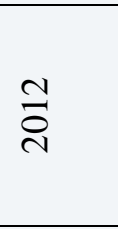 & $\begin{array}{l}\text { As possíveis inter-relações das } \\
\text { redes comunicativas - Blogs e das } \\
\text { comunidades de prática no } \\
\text { processo de formação de } \\
\text { professores de Matemática }\end{array}$ & $\begin{array}{lr}\text { Oliveira, } & \text { Maria } \\
\text { Angela } & \text { De } \\
\text { Oliveira } & \end{array}$ & $\begin{array}{l}\text { Dissertação } \\
\text { D2 }\end{array}$ & UNESP \\
\hline 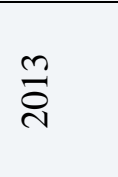 & $\begin{array}{l}\text { Comunidades de prática online: } \\
\text { contribuições à formação de } \\
\text { professores no Brasil e em } \\
\text { Portugal }\end{array}$ & $\begin{array}{l}\text { Silva, Flaviana } \\
\text { Dos Santos }\end{array}$ & $\begin{array}{l}\text { Tese } \\
\text { T1 }\end{array}$ & PUC-SP \\
\hline$\stackrel{\text { ¿ }}{\circ}$ & $\begin{array}{l}\text { A abordagem da Natureza da } \\
\text { Ciência subsidiada por recursos } \\
\text { fílmicos em uma Comunidade de } \\
\text { Prática }\end{array}$ & $\begin{array}{ll}\text { Santana, } & \text { Edson } \\
\text { Rodrigues } & \end{array}$ & $\begin{array}{l}\text { Tese } \\
\text { T2 }\end{array}$ & USP \\
\hline$\frac{n}{2}$ & $\begin{array}{l}\text { Ação e raciocínio pedagógico de } \\
\text { professoras de enfermagem em } \\
\text { diferentes contextos educacionais }\end{array}$ & $\begin{array}{l}\text { Menegaz, } \\
\text { Jouhanna } \\
\text { Carmo }\end{array}$ & $\begin{array}{c}\text { Tese } \\
\text { T3 }\end{array}$ & UFSC \\
\hline$\frac{n}{i}$ & $\begin{array}{l}\text { Os estágios supervisionados e } \\
\text { prática de ensino à luz das novas } \\
\text { diretrizes curriculares nacionais } \\
\text { para formação inicial e continuada } \\
\text { de professores da Educação Básica }\end{array}$ & $\begin{array}{l}\text { Matias, Aluska Da } \\
\text { Silva }\end{array}$ & $\begin{array}{l}\text { Dissertação } \\
\text { D3 }\end{array}$ & UEPB \\
\hline
\end{tabular}




\begin{tabular}{|c|c|c|c|c|}
\hline$\frac{0}{2}$ & $\begin{array}{l}\text { Formação docente no estágio não } \\
\text { obrigatório a partir de uma } \\
\text { "comunidade de prática" }\end{array}$ & $\begin{array}{ll}\text { Prates, } & \text { Soraia } \\
\text { Carise } & \end{array}$ & $\begin{array}{c}\text { Tese } \\
\text { T4 }\end{array}$ & PUC-PR \\
\hline 울 & $\begin{array}{l}\text { Comunidade de Prática na } \\
\text { Perspectiva da Inclusão Escolar: o } \\
\text { Perfil, os Discursos e as Práticas de } \\
\text { Educadores no Exercício da } \\
\text { Cultura da Participação }\end{array}$ & $\begin{array}{l}\text { Schneider, } \\
\text { Fernanda Chagas }\end{array}$ & $\begin{array}{l}\text { Tese } \\
\text { T5 }\end{array}$ & UFRGS \\
\hline 웅 & $\begin{array}{l}\text { Formação continuada na } \\
\text { perspectiva colaborativa para } \\
\text { professores que atuam com alunos } \\
\text { público-alvo da educação especial }\end{array}$ & $\begin{array}{l}\text { Peixinho, Marcia } \\
\text { Alexandra Araujo }\end{array}$ & $\begin{array}{l}\text { Dissertação } \\
\text { D4 }\end{array}$ & UESC \\
\hline 호 & $\begin{array}{l}\text { Proposta de um processo } \\
\text { colaborativo na plataforma moodle } \\
\text { para a aprendizagem de técnicas de } \\
\text { síntese sonora }\end{array}$ & $\begin{array}{l}\text { Ratton, Miguel } \\
\text { Balloussier }\end{array}$ & $\begin{array}{l}\text { Dissertação } \\
\text { D5 }\end{array}$ & UNINTER \\
\hline 혹 & $\begin{array}{l}\text { Um estudo com professores } \\
\text { supervisores do pibid sobre suas } \\
\text { compreensões/ações } \\
\begin{array}{llr}\text { avaliação acerca da } \\
\text { aprendizagem }\end{array} \\
\end{array}$ & $\begin{array}{l}\text { Oliveira, Luciani } \\
\text { De }\end{array}$ & $\begin{array}{l}\text { Dissertação } \\
\text { D6 }\end{array}$ & UNIOESTE \\
\hline 홍 & $\begin{array}{l}\text { Aprender a ensinar matemática: a } \\
\text { participação de estudantes de } \\
\text { pedagogia em uma comunidade de } \\
\text { prática }\end{array}$ & $\begin{array}{l}\text { Lacerda, Sara } \\
\text { Miranda De }\end{array}$ & $\begin{array}{c}\text { Tese } \\
\text { T6 }\end{array}$ & PUC-SP \\
\hline 혹 & $\begin{array}{l}\text { Teacher development in a } \\
\text { Community of practice in } \\
\text { Southern Brazil }\end{array}$ & Kirsch, William & $\begin{array}{c}\text { Tese } \\
\text { T7 }\end{array}$ & UFRGS \\
\hline 호 & $\begin{array}{l}\text { A formação de professores em } \\
\text { comunidades de prática por meio } \\
\text { da participação no pibid de } \\
\text { química em instituições de ensino } \\
\text { superior no estado do rio grande do } \\
\text { sul }\end{array}$ & $\begin{array}{l}\text { Ribeiro, Marcus } \\
\text { Eduardo Maciel }\end{array}$ & $\begin{array}{c}\text { Tese } \\
\text { T8 }\end{array}$ & PUC-RS \\
\hline 혼 & $\begin{array}{l}\text { Análise de um Grupo de Prática de } \\
\text { Professorxs que Ensinam } \\
\text { Matemática: Aspectos do } \\
\text { Desenvolvimento Profissional }\end{array}$ & $\begin{array}{l}\text { Silva, Fabio } \\
\text { Menezes Da }\end{array}$ & $\begin{array}{l}\text { Dissertação } \\
\text { D7 }\end{array}$ & UFRJ \\
\hline 호 & $\begin{array}{l}\text { Grupo colaborativo como } \\
\text { estratégia formativa de articulação } \\
\text { da educação infantil com o ensino } \\
\text { fundamental I }\end{array}$ & $\begin{array}{l}\text { Oliveira, Adriana } \\
\text { Beatriz De }\end{array}$ & $\begin{array}{l}\text { Dissertação } \\
\text { D8 }\end{array}$ & PUC-SP \\
\hline$\stackrel{\infty}{\stackrel{\sim}{\sim}}$ & $\begin{array}{l}\text { Unindo forças: reflexões sobre a } \\
\text { questão racial na construção } \\
\text { identitária no processo de } \\
\text { formação docente }\end{array}$ & $\begin{array}{lr}\text { Santos, } & \text { Raquel } \\
\text { Ferreira } & \text { Sampaio } \\
\text { Dos } & \end{array}$ & $\begin{array}{c}\text { Dissertação } \\
\text { D9 }\end{array}$ & UERJ \\
\hline$\frac{\infty}{\stackrel{\lambda}{\sim}}$ & $\begin{array}{l}\text { O professor enfermeiro novato da } \\
\text { educação profissional técnica de } \\
\text { nível médio e o conhecimento } \\
\text { pedagógico do conteúdo de lee } \\
\text { shulman }\end{array}$ & $\begin{array}{l}\text { Souza, Daniela } \\
\text { Maysa De }\end{array}$ & $\begin{array}{l}\text { Tese } \\
\text { T9 }\end{array}$ & UFSC \\
\hline
\end{tabular}




\begin{tabular}{|c|c|c|c|c|}
\hline$\frac{\infty}{\infty}$ & $\begin{array}{l}\text { Desenvolvimento de comunidade } \\
\text { de prática docente virtual: } \\
\text { possibilidade de formação do } \\
\text { enfermeiro docente novato }\end{array}$ & $\begin{array}{l}\text { Oliveira, Maria } \\
\text { Luiza Carvalho } \\
\text { De }\end{array}$ & $\begin{array}{l}\text { Tese } \\
\text { T10 }\end{array}$ & UFSC \\
\hline$\frac{\infty}{\infty}$ & $\begin{array}{l}\text { Um estudo sobre o ensino dos } \\
\text { temas "origem da vida" e } \\
\text { "evolução biológica" em } \\
\text { dissertações e teses brasileiras } \\
(2006 \text { a 2016) }\end{array}$ & Zabotti, Kamilla & $\begin{array}{l}\text { Dissertação } \\
\text { D10 }\end{array}$ & UNIOESTE \\
\hline$\frac{\infty}{0}$ & $\begin{array}{l}\text { A formação continuada de } \\
\text { professores para uso pedagógico } \\
\text { de Tecnologias Digitais de } \\
\text { Informação, Comunicação e } \\
\text { Expressão por meio de uma } \\
\text { comunidade de prática: Um estudo } \\
\text { de caso em torno do Grupo de } \\
\text { Educadores Google }\end{array}$ & $\begin{array}{l}\text { Oliveira, André } \\
\text { Luiz Pereira De }\end{array}$ & $\begin{array}{l}\text { Dissertação } \\
\text { D11 }\end{array}$ & UNB \\
\hline
\end{tabular}

Fonte: Elaborado pelos autores (2019)

Para facilitar o reconhecimento, as pesquisas foram identificadas, sendo as dissertações, através das iniciais D1, D2, D3, D4 até a D11 e as teses através das iniciais T1, T2, T3 até T10. No quadro 2 apresentamos uma síntese quantitativa de teses e dissertações selecionadas para a análise de dados. Destacamos que, os programas de pós-graduação da Universidade Federal de Santa Catarina - UFSC e a Pontifícia Universidade Católica de São Paulo são as instituições que possuem mais pesquisas acerca do tema investigado, com 3 produções cada, seguidas da Universidade Federal do Rio Grande do Sul - UFRGS e a Universidade Estadual do Oeste do Paraná - UNIOESTE com 2 produções respectivamente. Evidenciamos ainda que o tema é relevante para diversas regiões do país, sendo a região Sul com maior destaque, contribuindo com 11 pesquisas, seguido da região Sudeste que conta com 8 pesquisas.

Quadro 2 - Identificação das pesquisas selecionadas

\begin{tabular}{|c|c|c|}
\hline ANO & TESES & DISSERTAÇÕES \\
\hline $\mathbf{2 0 1 0}$ & 0 & 1 \\
\hline $\mathbf{2 0 1 2}$ & 0 & 1 \\
\hline $\mathbf{2 0 1 3}$ & 1 & 0 \\
\hline $\mathbf{2 0 1 4}$ & 1 & 0 \\
\hline $\mathbf{2 0 1 5}$ & 1 & 1 \\
\hline $\mathbf{2 0 1 6}$ & 2 & 1 \\
\hline $\mathbf{2 0 1 7}$ & 3 & 4 \\
\hline $\mathbf{2 0 1 8}$ & 2 & 3 \\
\hline SUBTOTAL & 10 & 11 \\
\hline TOTAL & & $\mathbf{2 1}$ \\
\hline
\end{tabular}

Fonte: Elaborado pelos autores (2019) 
A área da Educação, por meio do aumento contínuo das ofertas de cursos de pósgraduação, compõe uma das áreas em que houve um aumento significativo da produção científica. Devido a essa expansão, faz-se necessário que haja pesquisas de mapeamento que possibilitem analisar categorias que emergem diante desse contexto. Para Vosgerau e Romanowski (2014), esses estudos favorecem examinar as contribuições das pesquisas, na perspectiva da definição da área, do campo e das disciplinas que o compõem.

Nesta revisão temos como finalidade delimitar, clarificar e caracterizar o objeto de estudo por meio de levantamento bibliográfico seletivo restrito com parâmetros delimitados pelas pesquisadoras.

\section{ANÁLISE DOS DADOS}

A análise dos dados foi realizada partindo das categorias das pesquisas que foram préselecionadas, sendo elas: as temáticas pesquisadas, os objetivos de cada pesquisa, os principais autores utilizados bem como os resultados obtidos.

\subsection{Temáticas pesquisadas}

Na categoria temáticas pesquisadas, percebemos que há uma grande variedade de temas correlacionados. Pode-se verificar no quadro 3, a síntese das temáticas privilegiadas nas pesquisas.

Quadro 3: Temáticas privilegiada nas pesquisas selecionadas

\begin{tabular}{|c|c|c|c|}
\hline $\begin{array}{lll}\text { TEMÁTICAS } & \text { PRIVILEGIADAS } & \text { NAS } \\
\text { PESQUISAS } & \end{array}$ & TESES & DISSERTAÇÕES & TOTAL \\
\hline $\begin{array}{l}\text { Estágio supervisionado }- \\
\text { obrigatório }\end{array}$ & $\mathbf{1}$ & 1 & 2 \\
\hline $\begin{array}{l}\text { Estudos coletivos de casos }- \text { Relatos de } \\
\text { experiência }\end{array}$ & 1 & 1 & 2 \\
\hline Formação de professores - Ciências & $\mathbf{1}$ & $\mathbf{1}$ & 2 \\
\hline $\begin{array}{l}\text { Formação de professores }- \text { curso de } \\
\text { enfermagem }\end{array}$ & 2 & - & 2 \\
\hline Formação de professores - Educação inclusiva & $\mathbf{1}$ & 1 & 2 \\
\hline Formação de professores - Língua estrangeira & 1 & 2 & 3 \\
\hline Formação de professores - Matemática & - & 2 & 2 \\
\hline Formação de professores - Química & 1 & - & 1 \\
\hline Formação de professores em serviço & 1 & - & 1 \\
\hline Formação identitária do professor & - & 1 & 1 \\
\hline Formação Inicial e continuada - Pedagogia & 1 & 2 & 3 \\
\hline
\end{tabular}




\begin{tabular}{|l|l|l|l|}
\hline Grupo de Estudos na Cultura Digital & 1 & 1 & 2 \\
\hline PIBID & $\mathbf{1}$ & $\mathbf{1}$ & 2 \\
\hline Processos colaborativos & - & 4 & 4 \\
\hline Tecnologias da informação e comunicação - & $\mathbf{1}$ & $\mathbf{3}$ & 4 \\
\hline Ferramentas tecnológicas para a prática docente & & & \\
\hline
\end{tabular}

Fonte: Elaborado pelas autoras (2019)

Inferimos que as pesquisas em educação que tratam da formação inicial ou continuada de professores, envolvendo comunidades de prática, ocorrem nos mais diversos cursos e processos. Há pesquisas na área de Língua Estrangeira, Matemática, Ciências, entre outros. Isso possibilita que sejam contempladas uma gama ampla de temáticas. Podemos perceber que a relação entre as comunidades de práticas com processos de formação colaborativos e o uso das tecnologias da informação e comunicação como ferramenta tecnológica para a prática e a formação docente é uma das temáticas que mais surgiu nas discussões.

As contribuições das pesquisas nos dizem sobre a importância de investir em inovações, e aqui falamos em inovação no sentido que traz Gatti et al (2019): as inovações podem ser consideradas novas ou originais no lugar em que elas estão incorporadas, ou seja, algo é inovador em um determinado contexto e momento histórico.

Notamos que há um olhar atento para as inovações na educação desde a formação inicial, contemplando iniciativas como o PIBID ${ }^{1}$, o estágio supervisionado e o estágio não obrigatório, até a formação continuada, valorizando aspectos identitários dos docentes e os espaços de formação.

Outro aspecto abordado nas temáticas diz respeito à perspectiva colaborativa nos processos de formação. As pesquisas selecionadas dão ênfase ao processo de desenvolvimento profissional docente que acontece no coletivo seja ele no mesmo grupo escolar, disciplina ou área de formação e atuação, fortalece a troca de experiências, processos de auto confrontação, reflexão e o protagonismo profissional, propiciando e fortalecendo os processos de formação identitária. Podemos verificar tais aspectos na pesquisa D4, que traz a pesquisa colaborativa como importante impulsionadora do desenvolvimento profissional docente na perspectiva da educação inclusiva. Na pesquisa D7 do pesquisador Silva (2017), há uma ênfase nos aspectos do desenvolvimento profissional docente do profissional de matemática, olhando para a prática colaborativa de um grupo participante de uma CoP. Já a pesquisadora Oliveira (2017) reflete

\footnotetext{
${ }^{1}$ O PIBID é uma ação da Política Nacional de Formação de Professores do Ministério da Educação (MEC) que visa proporcionar aos discentes na primeira metade do curso de licenciatura uma aproximação prática com o cotidiano das escolas públicas de educação básica e com o contexto em que elas estão inseridas.
} 
como um grupo colaborativo, através de uma $\mathrm{CoP}$ pode ser visto como estratégia formativa na articulação entre a educação infantil e o ensino fundamental I. Ainda, na pesquisa D9, Santos (2018), contribui significativamente ao refletir questões de construção identitária nos processos de formação docente.

Ao encontro desse aspecto, surge a temática da Comunidade de Prática trazida pelas pesquisas selecionadas, como uma alternativa atual para colaborar na formação inicial e continuada na perspectiva coletiva, valorizando o grupo e contemplando outra temática correlacionada nas pesquisas que é a valorização da prática docente tanto para contemplar problemáticas reais quanto para pensar em formação diminuindo a distância entre teoria e prática.

A inovação abordada nas pesquisas reflete sobre os ambientes de formação docente, onde surge então a temática das tecnologias de informação e comunicação, uma importante aliada da contemporaneidade que propicia uma diversificada gama de instrumentos para a formação das comunidades de prática. Seguindo o mesmo raciocínio, Silva (2013), na Tese T1, compartilha os resultados de sua investigação, que evidenciou que o compartilhamento de experiências em uma CoP online permitiu construir conhecimento sobre a integração das TIC no contexto educacional. Os participantes passaram a compreender melhor o seu papel enquanto agentes de mudanças que planificam e refletem sobre suas ações pedagógicas em sala de aula. O uso das CoPs é ratificado pelo autor Santana (2014), que em sua tese - T2 sustenta que os mecanismos de autonomia e protagonismo da ação do professor foram contemplados em virtude da participação em uma $\mathrm{CoP}$, que valoriza e estimula o compartilhamento entre indivíduos que se identificam em práticas e ideias.

Neste sentido, inferimos que a Comunidade de Prática se configura em uma estratégia de formação inicial e continuada de professores que se adapta à necessidade de cada coletivo, podendo ser configurada considerando as práticas docentes e a realidade de cada contexto onde ela é desenvolvida.

\subsection{Objetivos das pesquisas}

Definir objetivos é parte essencial de uma pesquisa. É através deles que o pesquisador demonstra qual a sua intenção de pesquisa e quais caminhos teóricos e metodológicos serão traçados para obter os resultados ou considerações finais. Dentre as pesquisas analisadas para este artigo, considerando as temáticas, podemos afirmar que os objetivos são diversos, no 
entanto, há pontos comuns que devem ser considerados. Os objetivos verificados nas pesquisas selecionadas foram listados no quadro 4. É importante destacar que, os objetivos analisados são objetivos gerais das pesquisas.

Quadro 4: Objetivos privilegiados nas pesquisas selecionadas

\begin{tabular}{|l|c|c|c|}
\hline $\begin{array}{l}\text { OBJETIVOS PRIVILEGIADOS } \\
\text { NAS PESQUISAS }\end{array}$ & TESES & DISSERTAÇÕES & TOTAL \\
\hline Ampliar & - & 1 & 1 \\
\hline Analisar & 2 & 3 & 5 \\
\hline Compreender & 3 & - & 3 \\
\hline Conhecer & 2 & - & 2 \\
\hline Construir & 2 & - & 2 \\
\hline Descrever & 1 & 1 & 2 \\
\hline Desenvolver & 1 & 1 & 2 \\
\hline Discutir & 1 & 2 & 3 \\
\hline Ensinar & 1 & - & 1 \\
\hline Evidenciar & - & 2 & 2 \\
\hline Identificar & 1 & 1 & 2 \\
\hline Investigar & 1 & 2 & 3 \\
\hline Observar & 1 & - & 1 \\
\hline Relacionar & 1 & - & 1 \\
\hline Verificar & - & 1 & 1 \\
\hline
\end{tabular}

Fonte: Elaborado pelas autoras (2019)

À luz do artigo de Priscila Larocca et al (2015) intitulado Pesquisas sobre Formação de Professores no Mestrado da UEPG: uma análise dos objetivos expressos nas dissertações (1996 - 2013), analisamos os objetivos das pesquisas selecionadas nessa revisão sistemática. As autoras desenvolveram reflexões acerca dos objetivos nas pesquisas em educação e criaram uma tipologia que os caracterizam.

Larocca, Rosso e Souza (2005) classificam os objetivos em seis categorias, sendo elas: objetivos compreensivos, objetivos avaliativos, objetivos propositivos, objetivos descritivos, objetivos meios e objetivos generalistas. Os objetivos compreensivos contribuem para a captação de uma determinada realidade ou problema mais amplo. Já os objetivos avaliativos têm finalidade apreciativa e valorativa. Os objetivos propositivos indicam a necessidade de transformação em determinadas situações e instigam a criação de projetos ou ações para fins dessas modificações. Quando tratamos dos objetivos descritivos, não há quaisquer avaliações com relação ao material descrito, esses objetivos tratam especificamente da descrição, explanação detalhada de experiências, registros ou outro. Os objetivos meios, são ações inerentes à pesquisa, não visam responder à problematização, mas sim sustentá-la. E, por fim, 
os objetivos generalistas, possuem uma exagerada amplitude, o que inclusive, dificulta a percepção da temática ou problemática a que se referem.

Diante do exposto, organizamos os objetivos encontrados nas 21 teses ou dissertações, de acordo com a tipologia acima descrita, tendo chegado ao resultado expresso no quadro 5.

Quadro 5: Tipologia dos objetivos privilegiados nas pesquisas selecionadas

\section{TIPOLOGIA DOS OBJETIVOS PRIVILEGIADOS NAS PESQUISAS}

Objetivos Compreensivos

Compreender, Conhecer

Objetivos Avaliativos

Verificar, Relacionar

Objetivos Propositivos

Construir, Desenvolver, Ampliar, Ensinar

Objetivos Descritivos

Descrever, Identificar, Evidenciar

Objetivos Meio

Observar, Discutir

Objetivos Generalistas

Investigar, Analisar

Fonte: Elaborado pelas autoras (2019)

Percebe-se que os objetivos privilegiados nas teses e dissertações, classificam-se em sua maioria como objetivos propositivo e descritivos A tarefa de construção dos objetivos de pesquisa é, pois, um caminho a percorrer para auxiliar o sujeito pesquisador a apropriar-se do conhecimento e apreender seu objeto de pesquisa (LAROCCA et al, 2015). Analisar esses objetivos revela as principais características das pesquisas na área da educação e os percursos investigativos adotados nos dez anos selecionados.

\subsection{Principais autores pesquisados}

As pesquisas analisadas chamam a atenção para situar o leitor no contexto teórico do trabalho. Para maior esclarecimento, sustentação ou ilustração do assunto, foram selecionados os autores que embasaram os conceitos discutidos.

Ilustramos através do quadro 6, os autores citados nas pesquisas, que compreendem seu suporte teórico.

Quadro 6: Principais autores privilegiados nas pesquisas selecionadas

\begin{tabular}{l|l|l}
\multicolumn{2}{c}{ PRINCIPAIS AUTORES PRIVILEGIADOS NAS PESQUISAS } \\
$\begin{array}{c}\text { CONCEITO } \\
\text { APROPRIADO }\end{array}$ & \multicolumn{1}{|c}{ SUPORTE TEÓRICO } & \multicolumn{1}{c}{ PESQUISAS } \\
APrendizagem online & $\begin{array}{l}\text { Miskulin (2009), Miskulin et al (2011), Pallof e } \\
\text { Pratt (2004), Silva e Miskulin (2010). }\end{array}$ & D2. \\
m comunidades & $\begin{array}{l}\text { Wenger (1998) e Wenger, McDemott e Snyder } \\
\text { (2002), Wenger (2001). }\end{array}$ & $\begin{array}{l}\text { D1, D2, D5, D7, } \\
\text { D10, T1, T3, T4, } \\
\text { T5, T6, T7, T10. }\end{array}$ \\
\hline
\end{tabular}


Formação de professores

Formação de professores - Ciências

Formação de professores

- Matemática

Tecnologias

Informação

Comunicação

Educação

na
Alarcão (2003), Baldini (2014), Capellinni (2004), Desgagné (1998), Espinoza (2002), Freire (1987, 1996, 2001, 2011), Gatti (2009, 2010 2011), Giroux (1997), Ibiapina (2008), Imbernón (2001, 2009), Lago (2014), Masetto (2000), Mendes (2007; 2011), Mendes, Vilaronga e Zerbato (2014), Nóvoa (1988, 1991, 1992, 2009), Rabelo (2012), Sacristán (1991, 1998, 2002), Tardiff (2010), Valadão (2014), Zeichner (1993).

Acevedo (2010), Araújo Neto (2003), Bell, Lederman e Abd El-Khalick (1998), Borges (2007), Buaraphan (2009), Gil-Pérez Et Al (2001), Matthews (1995), Santana E Arroio (2009).

Almouloud (1992, 1997, 2007), D`Ambrosio (1993).

de Almeida (2000, 2002, 2004, 2005, 2008 2009, 2011, 2013), Fernandes (2007), Jorge Megid Neto (1999), Massetto (2000), Miskulin (2008), Moran (2000), Oliveira, 2011, Penteado (2004), Valente (1999)

D2, D4, D6, D7,

D8, D9, T1, T2, T3, T4, T5, T7, $\mathrm{T} 8$.

$\mathrm{T} 2$.

D2, T1.

D2, D11, T1, T2.

Fonte: Elaborado pelas autoras (2019)

As pesquisas selecionadas, de acordo com os critérios de busca, resultaram em 21 pesquisas, as quais analisamos a sustentação teórica que embasa cada discussão proposta. Um dos descritores para seleção de pesquisas trata das Comunidades de Prática, neste quesito verificamos que os autores Wenger (1998) e Wenger, McDemott e Snyder (2002), Wenger (2001) ganham destaque, pois foram mencionados nas pesquisas D1, D2, D5, D7, D10 e T1, T3, T4, T5, T6, T7 e T10.

Na sequência, há uma gama significativa de discussões que tratam da formação de professores. Os autores se apoiam em Alarcão (2003), Baldini (2014), Capellinni (2004), Desgagné (1998), Espinoza (2002), Freire (1987, 1996, 2001, 2011), Gatti (2009, 2010 2011), Giroux (1997), Ibiapina (2008), Imbernón (2001, 2009), Lago (2014), Masetto (2000), Mendes (2007; 2011), Mendes, Vilaronga e Zerbato (2014), Nóvoa (1988, 1991, 1992, 2009), Rabelo (2012), Sacristán (1991, 1998, 2002), Tardiff (2010), Valadão (2014) e Zeichner (1993) para as discussões acerca desse campo. No arcabouço teórico que envolve a Comunidade de Prática como instrumento da formação continuada, derivam outros autores que tratam de áreas específicas como, por exemplo Acevedo (2010), Araújo Neto (2003), Bell, Lederman e Abd El-Khalick (1998), Borges (2007), Buaraphan (2009), Gil-Pérez Et Al (2001), Matthews (1995), Santana e Arroio (2009) que discutem a formação de professores de ciências e Almouloud (1992, 1997, 2007), D`Ambrosio (1993) para a formação de professores de matemática. 
Em terceiro lugar, ganham destaque os autores que tratam da tecnologia de comunicação e informação vinculados à educação, sendo Almeida (2000, 2002, 2004, 2005, 2008 2009, 2011, 2013), Fernandes (2007), Jorge Megid Neto (1999), Marcelo Teixeira (2008), Mário Oliveira (2011), Massetto (2000), Miskulin (2008), Moran (2000), Oliveira, 2011, Penteado (2004), Valente (1999) citados nas pesquisas D2, D11, T1 e T2.

Grande parte das propostas de pesquisa, tratam do uso das CoPs online, por meio de ferramentas tecnológicas que envolvem os professores e a formação continuada em novos espaços, contribuindo e trazendo inovação para o tema. Neste sentido importa observarmos os principais resultados obtidos mediante as discussões supracitadas.

\subsection{Resultados obtidos}

Para além de observar as temáticas, os objetivos de pesquisa e os principais autores privilegiados nas teses e dissertações selecionadas, entendemos como de fundamental importância um olhar para os resultados alcançados. Isso porque, é através dos resultados que verificamos quais os caminhos que têm contribuído de maneira significativa na trajetória da pesquisa educacional, auxiliando a identificação de lacunas e oportunidades de novas pesquisas, bem como as inovações e caminhos que vem sendo trilhados por pesquisadores da área. Os resultados das pesquisas podem ser analisados no quadro 7.

\section{Quadro 7: Principais resultados obtidos nas pesquisas selecionadas}

\section{PRINCIPAIS RESULTADOS OBTIDOS}

D1 Importância do planejamento e de conhecer a necessidade do grupo.

D2 Reflete sobre as potencialidades das CoPs online, que ampliam os horizontes proporcionando trocas e desenvolvimento de aprendizagens sem os limites geográficos.

D3 Os resultados deste trabalho apontaram várias questões que precisam ser debatidas para recomposição dos Projetos Pedagógicos de Cursos à luz das novas DCNs para formação inicial e continuada de professores da Educação Básica.

D4 Identificou a necessidade de uma melhor integração e parceria entre os professores das SEM (Salas de Recursos Multifuncionais) e salas comuns e considera o uso das CoPs uma parceria que pode ser concretizada por meio de um trabalho de formação continuada na perspectiva colaborativa o que reflete no trabalho docente e melhora o desempenho, a aprendizagem e escolarização de alunos PAEE (Público Alvo da Educação Especial).

D5 A implementação de uma CoP para ensino à distância precisa ser muito bem concebida e elaborada. O suposto poder da aprendizagem a distância depende muito da combinação adequada da tecnologia com o processo pedagógico proposto, de maneira que se isso não for bem articulado corre-se o risco de não se aproveitar todo o potencial imaginado e, até mesmo, chegar-se a um resultado contraproducente.

D6 Conclui que é importante discutir a avaliação do ensino e da aprendizagem dentro dos cursos de licenciatura, bem como dos subprojetos do Programa Institucional de Bolsas de Iniciação à Docência. 
D7 O N-LSM mostrou-nos inicialmente ser um grupo escolar, aberto à prática colaborativa $\mathrm{e}$ à parceria com universidades, mas que não deseja ser colonizado por essas instituições. Suas práticas, modos de interação, compartilhamento e articulação de saberes, anseios e produção de conhecimentos, trazem também características que permitem serem chamadas de uma comunidade de prática.

D8 Os resultados revelaram que o trabalho colaborativo se constitui em uma estratégia eficaz de reflexão e aprimoramento da prática docente ao contribuir com os sujeitos participantes na construção de um caminho que considere as principais demandas locais, dando maior visibilidade e compreensão entre o Currículo prescrito e o praticado, por meio das negociações entre as participantes do processo que passaram a investigar as próprias ações se constituindo em uma comunidade de prática.

D9 Defende que uma CoP pode ser uma estratégia para construir significados referentes as nossas identidades, em especial, à identidade de professores negros de inglês.

D10 Os resultados desta pesquisa, bem como a realização de pesquisas dedicadas à análise do conjunto da produção acadêmica, podem contribuir para a implantação de propostas mais específicas na formação de professores e melhorias no ensino e na aprendizagem da Origem da Vida e Evolução Biológica.

D11 Ao longo desta pesquisa, foi possível perceber que as comunidades são espaços que concentram pessoas realmente apaixonadas por algum tema, a lição mais importante é que as pessoas e as interações entre elas ainda representam a mais valiosa tecnologia para a educação.

T1 Os resultados evidenciaram que o compartilhamento de experiência em uma COP online permitiu construir conhecimento sobre a integração das TDIC no contexto educacional. Os participantes passaram a compreender melhor o seu papel enquanto agente de mudanças que planifica e reflete sobre suas ações pedagógicas em sala de aula.

T2 Os mecanismos de autonomia e protagonismo da ação do professor foram contemplados em virtude da participação em CoP, que valoriza e estimula o compartilhamento entre indivíduos que se identificam em práticas e ideias.

T3 Houve o entendimento do contexto como espaço de aprendizagem da prática docente e não somente como espaço de trabalho. As professoras foram inseridas em contextos diferentes de trabalho e com participações diferentes nestes contextos, consolidando participações distintas e aprendizagens distintas do que é ser professor. Esta ideia foi consolidada e corroborou para a confirmação da tese a partir da introdução do conceito de comunidades de prática.

T4 Defende a importância das reuniões de comunidade de prática para a formação docente dos estagiários

T5 Os resultados ainda indicam que iniciativas como a CPIE, podem adquirir uma conotação mais ligada à suplementação da formação docente, onde práticas desencadeadas a partir dos eixos de comunicação ou de resolução de problemas contribuem para que professores notadamente experientes sigam em contato, colaborando uns com os outros.

T6 Os resultados indicam que a participação na CoP OBEDUC possibilitou a reflexão e a interlocução com os pares, promovendo aprendizagem da docência tanto das estudantes como dos professores experientes, pela negociação de significados gerada no movimento dual de participação e retificação, pela constituição da identidade docente e pela prática.

T7 Os resultados apontam que a comunidade tem, tanto práticas formais, como práticas informais de formação de professores. As práticas formais são planejadas pela coordenadora e implementadas nas reuniões pedagógicas semanais, e incluem práticas como microteaching, workshops e palestras. As práticas informais emergem do dia a dia da comunidade, principalmente na sala dos professores e incluem práticas, tais como compartilhar materiais, pedir ajuda, compartilhar histórias de sala de aula, compartilhar bibliografia e conceitos teóricos e planejar aulas conjuntamente.

T8 A análise realizada das respostas produziu argumentos que sustentam a tese de que as ações realizadas no âmbito do Pibid atuam na forma de comunidades de prática, pois sua organização interna e seus resultados se equiparam com os pressupostos teóricos desse 


\begin{tabular}{|l|l|}
\hline T9 & $\begin{array}{l}\text { modo de ação formativa. Com isso, depreende-se que a participação de licenciandos no } \\
\text { Pibid desempenha papel fundamental na formação docente. }\end{array}$ \\
$\begin{array}{l}\text { As propostas de formação docente devem utilizar estratégias reflexivas sobre a própria } \\
\text { prática docente e sugere-se o uso de estratégias de mentoring, com observação da prática } \\
\text { dos colegas e aprendizagem horizontal com os pares (professores experientes), uso do } \\
\text { portfólio reflexivo como estratégia de avaliação formativa, criação de comunidade de } \\
\text { prática, lesson study e implantação do período de indução profissional, sendo para isto, } \\
\text { imprescindível o apoio institucional. }\end{array}$ \\
T10 & $\begin{array}{l}\text { As comunidades de prática docente na saúde e na enfermagem estão contribuindo para a } \\
\text { formação dos profissionais de saúde que seguem a carreira docente, especialmente para a } \\
\text { aquisição de conhecimentos pedagógicos. }\end{array}$ \\
\hline
\end{tabular}

Fonte: Elaborado pelas autoras (2019)

Notamos que nem todos os resultados apontam para a Comunidade de Prática, em consonância com os objetivos de cada pesquisa. Por isso, serão analisadas as pesquisas que tiveram como foco e mencionaram as CoPs.

A autora Bohn (2010) traz como resultado de sua dissertação - D1, a importância de um planejamento para a criação de um CoP. Sinaliza ainda que, é imprescindível conhecer a necessidade do grupo para então escolher ferramentas de tecnologia de comunicação que vão ao encontro do que ela intitula Desenho pedagógico. Essa afirmação vai ao encontro dos princípios defendidos pelo autor Wenger, verificando que quando respeitados os parâmetros de criação, uma comunidade de prática torna-se real e produtiva.

Referindo-se às TICs - Tecnologias de Informação e Comunicação em ambiente escolar, a autora Oliveira (2012), afirma em sua dissertação - D2, que os professores estão cientes dos desafios de inseri-las nas práticas docentes e que há muito que problematizar, refletir e experimentar para que a informação se transforme em conhecimento. Nesse sentido, reafirma a importância da formação continuada, pois as experiências proporcionadas por meio do Curso de Extensão, por ela proposto, propiciaram influências na prática dos professores, que se deixaram "tocar" pelas TIC. Aqui podemos observar a potencialidade das CoPs online, que ampliam os horizontes proporcionando trocas e desenvolvimento de aprendizagens sem os limites geográficos.

Prates (2016) discute na tese T4 que as CoPs são indicadas não somente para a formação continuada de professores, mas também na formação inicial. Com sua pesquisa, defende a importância das reuniões de comunidade de prática para a formação docente dos estagiários, uma vez que estes, ao participarem das reuniões, têm a oportunidade de aprender sobre a prática docente. Sugere ainda que os resultados obtidos podem contribuir para a conscientização de programas de formação docente e sobre a importância de projetos junto à 
escola que permitam que os estagiários trabalhem colaborativamente e atuem na prática de ensino.

Do mesmo modo, quando pensamos na formação de professores para a educação inclusiva e para professores da educação especial, as CoPs fomentam a reflexividade. Peixinho (2016) na sua dissertação - D4 contribui dizendo que essa parceria pode ser concretizada por meio de um trabalho de formação continuada na perspectiva colaborativa, visando contribuir com o trabalho desses profissionais para torná-los críticos e reflexivos acerca de suas práticas pedagógicas, melhorando assim o desempenho, a aprendizagem e escolarização de alunos PAEE - Público Alvo da Educação Especial.

Oliveira (2018) na tese - T10 confirma que a comunidade de prática docente promove uma aprendizagem situada na prática, facilita a reflexão sobre a prática do ensino, ajuda no desenvolvimento do conhecimento base para o ensino e no seu compartilhamento, constrói a identidade docente, fortalecendo o papel do docente, reduz o isolamento do docente e a prática da busca pela formação individualizada e, por isso é definida como uma ação de modificação da práxis docente.

\section{Considerações finais}

De acordo com a literatura selecionada para essa revisão sistemática, podemos afirmar que as propostas atuais relacionadas à formação de professores, indicam contribuições positivas nos processos de formação inicial e continuada que contemple a perspectiva coletiva e colaborativa. Do mesmo modo, processos como a comunidade de prática, tanto de maneira presencial como online, ampliam os espaços de reflexão, discussão, trocas de experiências, possibilitando a modificação da prática docente à luz de teorias.

Nessa direção, destacamos que na atualidade, onde a escola e os espaços de formação estão sendo acometidos pelos avanços da tecnologia de informação e comunicação, há possibilidades de junção, constituindo estratégias de desenvolvimento profissional docente, eficazes e modernas, através do uso de ferramentas tecnológicas e ressignificando espaços de formação e a própria identidade docente.

Por meio da análise de dados realizada nesse artigo é possível observar que as investigações na área da educação buscam uma evolução em harmonia com as novas Tecnologias de Informação e Comunicação, utilizando-se de diversas ferramentas que possibilitam maior interação entre professores desde sua formação inicial até a formação 
continuada. No entanto é preciso manter-se atento, pois nem sempre uma comunidade é uma comunidade de prática. As CoPs ultrapassam os limites organizacionais, sendo sua participação voluntária, não podendo ser equivalentes às networks informais ou comunidades de interesses comuns, cujo objetivo é apenas distribuir informações.

Verificamos ainda que, a partir dos resultados das pesquisas, o professor em nada perde quando se abre às novas possibilidades tecnológicas. A CoP utilizada em suas mais diversas possibilidades, desde presenciais ou online, corroborou com a troca de experiências entre profissionais auxiliando o desenvolvimento profissional, a reflexão de sua prática e até mesmo a autonomia pessoal para tomada de decisões mais conscientes diante da complexidade do dia a dia profissional. Desta maneira identificamos quanto a comunidade de prática pode ser uma ferramenta importante, quando bem planejada.

Constatamos ainda que uma CoP pode educar as pessoas a identificar os potenciais do trabalho coletivo e legitimar esse espaço, ajudando no enfrentamento de objetivos, favorecendo a confiança e o trabalho significativo, ampliando as possibilidades de compartilhamento de conhecimento, desenvolvimento pessoal, construção de identidades, bem como do acompanhamento de estratégias a curto, longo prazo ou novas estratégias.

\section{REFERÊNCIAS}

DE ALMEIDA, Maria Elizabeth Bianconcini. Tecnologias na Educação: dos caminhos trilhados aos atuais desafios. Boletim de Educação Matemática, v. 21, n. 29, p. 99-129, 2008.

BOGDAN, Robert; BIKLEN, Sari Knopp. Pesquisa qualitativa para educação: uma introdução à teoria e métodos, Boston: Allyn e Bacon, 1982.

BOHN, Vanessa Cristiane Rodrigues et al. Comunidades de prática na formação docente: aprendendo a usar ferramenta da web 2.0. 158f. Dissertação (Mestrado em Linguística aplicada). Universidade Federal de Minas Gerais, Belo Horizonte, 2010. Orientadora: Prof ${ }^{a}$ Dr $^{a}$ Vera Lúcia Menezes de Oliveira e Paiva.

BRASIL, Ministério da Educação. Base nacional comum curricular. Brasília-DF: MEC, Secretaria de Educação Básica, 2017. Disponível em:

<http://basenacionalcomum.mec.gov.br/images/BNCC_EI_EF_110518_versaofinal_site.pdf. Acesso em: 23 de agosto de 2019.

BRASIL. Ministério da Educação. Secretaria de Educação Básica. Resolução nº 02 de 01 de julho de 2015. Diretrizes Curriculares Nacionais para a formação inicial em nível superior e para a formação continuada. Brasília: MEC, SEB, 2015. Disponível em: < 
http://portal.mec.gov.br/docman/agosto-2017-pdf/70431-res-cne-cp-002-03072015-pdf/file>. Acesso em: 20 de agosto de 2019.

SILVA, Flaviana dos Santos. Comunidades de prática online: contribuições à formação de professores no Brasil e em Portugal.233f. Tese (Doutorado em Educação). Pontifícia Universidade Católica de São Paulo, São Paulo, 2013. Orientadora Prof ${ }^{a}$. Dr ${ }^{a}$ Maria Elizabeth Bianconini Trindade Pinto de Almeida.

GATTI, Bernadete Angelina; BARRETO, Elba Siqueira de Sá; ANDRÉ, Marli Eliza Dalmazo Afonso de; ALMEIDA, Patrícia Cristina Albieri de (Orgs). Professores do Brasil: novos cenários de formação. Brasília: UNESCO, 2019.

IMBERNÓN, Francisco. Formação docente e profissional: formar-se para a mudança e a incerteza/Francisco Imbernón; [tradução Silvana Cobucci Leite] - 9 ed. São Paulo: Cortez, 2011.

IMBERNÓN, Francisco. Formação permanente do professorado: novas tendências. $1^{\mathrm{a}}$. Ed. São Paulo. Cortez, 2009.

LAROCCA, Priscila; ROSSO, Ademir José; DE SOUZA, Audrey Pietrobelli. A formulação dos objetivos de pesquisa na pós-graduação em Educação: uma discussão necessária. Revista Brasileira de Pós-Graduação, v. 2, n. 3, 2005. Disponível em: < http://ojs.rbpg.capes.gov.br/index.php/rbpg/article/view/62> Acesso em: 2 de setembro de 2019.

NÓVOA, A. Formação de professores e profissão docente. In: Nóvoa, A. (Org.). Os professores e sua formação. Lisboa: Dom Quixote, 1992.

NÓVOA, António. Imagens do futuro presente. Lisboa: Educa, 2009.

OLIVEIRA, Maria Angela de Oliveira. As possíveis inter-relações das redes comunicativas blogs - e das comunidades de prática no processo de formação de professores de Matemática. 200 f. Dissertação (mestrado em Educação Matemática). Universidade Estadual Paulista, Instituto de Geociências e Ciências Exatas, Rio Claro, 2012. Disponível em: <http://hdl.handle.net/11449/91060>. Acesso em 2 de setembro de 2019. Orientadora Profa. Dra. Rosana Giaretta Sguerra Miskulin.

PEIXINHO, Márcia Alexandra Araújo. Formação continuada na perspectiva colaborativa para professores que atuam com alunos público-alvo da educação especial. 143f. Dissertação (mestrado em Educação Universidade Estadual de Santa Cruz, Ilheus, 2016. Orientadora: Prof ${ }^{a}$ Dra. Rosenaide Pereira dos Reis Ramos. Disponível em: < http://www.biblioteca.uesc.br/biblioteca/bdtd/201420645D.pdf> Acesso em: 2 de setembro de 2019.

ROMANOWSKI, Joana Paulin; ENS, Romilda Teodora. As pesquisas denominadas do tipo" estado da arte" em educação. Revista diálogo educacional, v. 6, n. 19, p. 37-50, 2006. Disponível em: https://www.redalyc.org/pdf/1891/189116275004.pdf. Acesso em: < 5 de agosto de 2019. 
SANTANA, Edson Rodrigues. A abordagem da natureza da ciência subsidiada por recursos fílmicos em uma comunidade de prática. 378 f. Tese (Doutorado em educação). Universidade de São Paulo, São Paulo, 2014. Orientador: Prof. Doutor Agnaldo Arroio.

SILVA, Fábio Menezes da. Análise de uma comunidade de prática de professorxs que ensinam matemática: aspectos do desenvolvimento profissional. 203f. Dissertação (Mestrado em Educação). Universidade Federal do Rio de Janeiro, Rio de Janeiro, 2017. Orientador: Prof. Doutor Victor Augusto Giraldo.

SNYDER, William; WENGER, Etienne; DE SOUSA BRIGGS, Xavier. Communities of practice in government: Leveraging knowledge for performance. Public Manager., v. 32, n. 4, p. 17-22, 2004. Disponível em:

https://www.researchgate.net/profile/William_Snyder3/publication/245706269_Communities _of_practice_in_government_Leveraging_knowledge_for_performance/links/54592d7f0cf26 d5090ad02b1.pdf. Acesso em: 27 de julho de 2019.

VOSGERAU, Dilmeire Sant'Anna Ramos; ROMANOWSKI, Joana Paulin. Estudos de revisão: implicações conceituais e metodológicas. Diálogo Educacional, v. 14, n. 41, p. 165189, 2014. Disponível em:

https://periodicos.pucpr.br/index.php/dialogoeducacional/article/view/2317. Acesso em: 22 de julho de 2019.

WENGER, Etienne. Cultivating communities of practice: a quick start-up guide for communities of practice. Disponível em: http://wenger-trayner.com/project/community-ofpractice-start-up-guide/. 2002. Acesso em: 22 de julho de 2019.

WENGER, Etienne. Communities of practice and social learning systems: the career of a concept. In: Social learning systems and communities of practice. Springer, London, 2010. p. 179-198.

WENGER-TRAYNER, Etienne; WENGER-TRAYNER, Beverly. Communities of practice: a brief introduction. 2015. Reference Source, 2016. Disponível em: < https://wengertrayner.com/introduction-to-communities-of-practice>. Acesso em: junho 2019.

WENGER, Etienne C.; MCDERMOTT, Richard; SNYDER, Williams C. of Practice: A Guide to Managing Knowledge, Harvard Business School Press, Cambridge, USA, 2002, 304 pages (ISBN 1-5781-330-8).

\section{SOBRE AS AUTORAS:}

\section{Greice Maiara Denker}

Mestranda em Educação, Universidade Regional de Blumenau (FURB) - Brasil; Programa de Pós-Graduação em Educação; GPFORPE - Grupo de Pesquisa em Formação de Professores e Práticas Educativas; E-mail: greice.denker@gmail.com

(iD https://orcid.org/0000-0002-9850-4324 


\section{Camila Galvan}

Mestranda em Educação, Universidade Regional de Blumenau (FURB) - Brasil; Programa de Pós-Graduação em Educação; GPFORPE - Grupo de Pesquisa em Formação de Professores e Práticas Educativas; E-mail: cgalvan@gmail.com

(iD http://orcid.org/0000-0002-1731-9363

\section{Rita Buzzi Rausch}

Doutorado (UNICAMP) e Pós-doutorado em Educação (UFSC), Professora Visitante no Programa de Pós-Graduação de Educação da Universidade Regional de Blumenau (FURB) e da Universidade da Região de Joinville (UNIVILLE) - Brasil; GPFORPE - Grupo de Pesquisa em Formação de Professores e Práticas Educativas. E-mail: ritabuzzirausch@ gmail.com

iD http://orcid.org/0000-0002-9413-4848 